PROCEEDINGS OF THE

AMERICAN MATHEMATICAL SOCIETY

Volume 131, Number 12, Pages 3783-3793

S 0002-9939(03)06940-5

Article electronically published on February 28, 2003

\title{
GLOBAL ANALYTIC REGULARITY FOR NON-LINEAR SECOND ORDER OPERATORS ON THE TORUS
}

\author{
CHIARA BOITI AND LUISA ZANGHIRATI \\ (Communicated by David S. Tartakoff)
}

\begin{abstract}
Assuming a subelliptic a-priori estimate we prove global analytic regularity for non-linear second order operators on a product of tori, using the method of majorant series.
\end{abstract}

\section{INTRODUCTION}

Hypoellipticity for linear partial differential operators has been largely investigated by many authors. In the non-linear case, on the contrary, there are still few results and many open questions.

Some results about $C^{\infty}$-hypoellipticity for non-linear partial differential equations have been obtained in $[\underline{\mathrm{X}}$ and $[\mathrm{G}]$, using the para-differential calculus of Bony [B].

We are interested in analytic hypoellipticity for non-linear second order p.d.e.'s. Local analytic regularity for a model operator given by sums of squares of non-linear vector fields has been proved in [TZ. Here we prove global analytic regularity on the torus for non-linear second order operators constructed from rigid vector fields, generalizing the result obtained for the linear case in $[\mathrm{T}]$.

The problem of regularity of solutions on the torus in the linear case has been studied by many other authors in the frameworks of $C^{\infty}$, Gevrey and analytic functions (see, for instance, GPY and the references there).

\section{Notation AND MAIN RESUlt}

Let $\mathbb{T}^{N}$ be the $N$-dimensional torus and split $\mathbb{T}^{N} \simeq \mathbb{T}^{m} \times \mathbb{T}^{n}$. Let us then consider, for $u \in C^{\infty}\left(\mathbb{T}^{N}\right)$ and for some integer $n^{\prime} \geq n$, the operator

$$
\begin{aligned}
P & =P_{u}=P(x, u, D) \\
& =\sum_{j, k=1}^{n^{\prime}} a_{j k}(u(t, x)) X_{j} X_{k}+\sum_{j=1}^{n^{\prime}} b_{j}(u(t, x)) X_{j}+X_{0}+c(u(t, x))
\end{aligned}
$$

Received by the editors July 4, 2002.

2000 Mathematics Subject Classification. Primary 35B65, 35B45; Secondary 35H10, 35H20.

Key words and phrases. Analytic regularity, non-linear, sums of squares of vector fields, torus. 
defined for $(t, x) \in \mathbb{T}^{m} \times \mathbb{T}^{n}$, where the real analytic coefficients $a_{j k}(u), b_{j}(u)$ and $c(u)$ are complex valued, but the real analytic rigid vector fields

$$
X_{j}=\sum_{k=1}^{n} d_{j k}(x) \frac{\partial}{\partial x_{k}}+\sum_{k=1}^{m} e_{j k}(x) \frac{\partial}{\partial t_{k}}, \quad j=0, \ldots, n^{\prime},
$$

are real valued (rigid means that the coefficients $d_{j k}, e_{j k}$ do not depend on $t$ ). Assume also that, for every $x \in \mathbb{T}^{n}$, the fields

$$
X_{j}^{\prime}=\sum_{k=1}^{n} d_{j k}(x) \frac{\partial}{\partial x_{k}}, \quad j=1, \ldots, n^{\prime},
$$

span the tangent space $T_{x}\left(\mathbb{T}^{n}\right)$.

Let us now denote by $A\left(\mathbb{T}^{N}\right)$ the space of real analytic functions on $\mathbb{T}^{N}$, and fix a solution $u \in C^{\infty}\left(\mathbb{T}^{N}\right)$ of the equation $P u=f$ for $f \in A\left(\mathbb{T}^{N}\right)$.

We shall assume in the sequel that the following a-priori estimate is satisfied for some $\delta, C>0$ and for all $v \in C^{\infty}\left(\mathbb{T}^{N}\right)$ :

$$
\sum_{i, j=1}^{n^{\prime}}\left\|X_{i} X_{j} v\right\|_{\mu}+\sum_{j=1}^{n^{\prime}}\left\|X_{j} v\right\|_{\mu}+\|v\|_{\mu+\delta} \leq C\left(\left\|P_{u} v\right\|_{\mu}+\|v\|_{\mu}\right),
$$

where $\mu$ is a fixed integer with $\mu>N / 2$, so that the Sobolev space $H^{\mu}\left(\mathbb{T}^{N}\right)$ is an algebra and

$$
\|f g\|_{\mu} \leq \Lambda\|f\|_{\mu} \cdot\|g\|_{\mu} \quad \forall f, g \in H^{\mu}\left(\mathbb{T}^{N}\right),
$$

for a positive $\Lambda$ depending only on $N$.

Before giving the analytic regularity result, we first give an example of an operator of type (11) satisfying the required assumptions, and in particular the a-priori estimate (4).

Example 2.1. Let $(t, x) \in \mathbb{T}^{2}$ and consider the operator

$$
P=\partial_{x}^{2}+\sin ^{2} x\left(1+a^{2}(u(t, x))\right) \partial_{t}^{2},
$$

where $a(u)$ is a real analytic function. This operator is of the form (11) with

$$
\begin{aligned}
& X_{1}=X_{1}^{\prime}=\partial_{x}, X_{2}=\sin x \partial_{t}, \\
& a_{11}(u) \equiv 1, a_{12}(u) \equiv a_{21}(u) \equiv 0, a_{22}(u)=1+a^{2}(u) .
\end{aligned}
$$

We must prove the a-priori estimate (4). From [RS] it easily follows that

$$
\|v\|_{\mu+\delta}^{2} \leq c\left(\sum_{j=1}^{2}\left\|X_{j} v\right\|_{\mu}^{2}+\|v\|_{\mu}^{2}\right) \quad \forall v \in C^{\infty}\left(\mathbb{T}^{2}\right), \delta=1 / 2 .
$$

This implies, by standard arguments, the following a-priori estimate for the operator $\tilde{P}=\partial_{x}^{2}+\sin ^{2} x \partial_{t}^{2}$ :

$$
\sum_{i, j=1}^{2}\left\|X_{i} X_{j} v\right\|_{\mu}^{2}+\sum_{j=1}^{2}\left\|X_{j} v\right\|_{\mu+\delta}^{2}+\|v\|_{\mu+2 \delta}^{2} \leq c^{\prime}\left|\langle\tilde{P} v, v\rangle_{\mu}\right|+\|v\|_{\mu}^{2},
$$

for some $c^{\prime}>0$ and for all $v \in C^{\infty}\left(\mathbb{T}^{2}\right)$. 
Since $P=\tilde{P}+a^{2}(u) X_{2}^{2}$, we have that

$$
\begin{aligned}
\left|\langle\tilde{P} v, v\rangle_{\mu}\right| & \leq\left|\langle P v, v\rangle_{\mu}\right|+\left|\left\langle a^{2}(u) X_{2}^{2} v, v\right\rangle_{\mu}\right| \\
& \leq \frac{1}{2}\|P v\|_{\mu}^{2}+\frac{1}{2}\|v\|_{\mu}^{2}+\varepsilon\left\|a^{2}(u) X_{2}^{2} v\right\|_{\mu}^{2}+\frac{1}{4 \varepsilon}\|v\|_{\mu}^{2} \\
& \leq \frac{1}{2}\|P v\|_{\mu}^{2}+\frac{2 \varepsilon+1}{4 \varepsilon}\|v\|_{\mu}^{2}+\varepsilon K\left\|X_{2}^{2} v\right\|_{\mu}^{2}
\end{aligned}
$$

for some constant $K>0$. Substituting in (5) we obtain the desired estimate (4), for $\varepsilon>0$ small enough.

Let us now state the main result of this paper.

Theorem 2.2. Let $P$ be the operator defined in (10), and assume that the vector fields $\left\{X_{j}\right\}_{j=0, \ldots, n^{\prime}}$ are rigid and that for every fixed $x \in \mathbb{T}^{n}$ the $\left\{X_{j}^{\prime}\right\}_{j=1, \ldots, n^{\prime}}$ span $T_{x}\left(\mathbb{T}^{n}\right)$.

Assume moreover that $u \in C^{\infty}\left(\mathbb{T}^{N}\right)$ is a solution of the equation

$$
P(x, u, D) u=f,
$$

for some $f \in A\left(\mathbb{T}^{N}\right)$, and that the a-priori estimate (4) is satisfied. Then also $u \in A\left(\mathbb{T}^{N}\right)$.

Remark 2.3. We can follow $[\mathrm{X}]$ to obtain from the a-priori estimate (4) and the use of para-differential operators a result of $C^{\infty}$-hypoellipticity for the operator (1) with the given assumptions on the $X_{j}$ 's: if $f \in C^{\infty}\left(\mathbb{T}^{N}\right)$ and $u \in C^{\mu+3}\left(\mathbb{T}^{N}\right)$ is a solution of $P u=f$, then $u \in C^{\infty}\left(\mathbb{T}^{N}\right)$.

Before giving the proof of Theorem 2.2, we first need some notation.

Define, for $u \in C^{\infty}\left(\mathbb{T}^{N}\right)$,

$$
\|u\|_{\mu}=\sum_{i, j=1}^{n^{\prime}}\left\|X_{i} X_{j} u\right\|_{\mu}+\sum_{j=1}^{n^{\prime}}\left\|X_{j} u\right\|_{\mu}+\|u\|_{\mu+\delta},
$$

and consider the sequence $m_{q}=c q ! /(q+1)^{2}$, where the constant $c$ is such that (see (AM])

$$
\sum_{0 \leq \beta \leq \alpha}\left(\begin{array}{c}
\alpha \\
\beta
\end{array}\right) m_{|\beta|} m_{|\alpha-\beta|} \leq m_{|\alpha|} .
$$

Then set $M_{q}=\varepsilon^{1-q} m_{q}$ for $\varepsilon>0$ and $q \geq 1$. The relation (6) implies that

$$
\sum_{0<\beta<\alpha}\left(\begin{array}{c}
\alpha \\
\beta
\end{array}\right) M_{|\beta|} M_{|\alpha-\beta|} \leq \varepsilon M_{|\alpha|}
$$

and hence, if we consider the formal power series

$$
\theta(Y)=\sum_{\alpha>0} \frac{M_{|\alpha|}}{\alpha !} Y^{\alpha}
$$

for $Y=(t, x) \in \mathbb{R}^{N}$, we obtain that

$$
\theta^{q}(Y) \ll \varepsilon^{q-1} \theta(Y) \quad \forall q \geq 1, Y \in \mathbb{R}^{N},
$$

meaning that each coefficient of the formal power series on the left is less than or equal to the corresponding coefficient of the formal power series on the right-hand side. 
It follows that, if we choose $A, R>0$ satisfying for every integer $q \geq 0$

(9) $\sum_{i, j=1}^{n^{\prime}}\left\|a_{i j}^{(q)}\right\|_{H^{\mu}\left(u\left(\mathbb{T}^{N}\right)\right)}+\sum_{j=1}^{n^{\prime}}\left\|b_{j}^{(q)}\right\|_{H^{\mu}\left(u\left(\mathbb{T}^{N}\right)\right)}+\left\|c^{(q)}\right\|_{H^{\mu}\left(u\left(\mathbb{T}^{N}\right)\right)} \leq A R^{q} q$ !

(which is possible because of the analyticity of the coefficients), and we define the formal power series

$$
\phi(w)=\sum_{q=1}^{+\infty} A R^{q} w^{q}, \quad \text { for } w \in \mathbb{R},
$$

then, for every $\rho>0$,

$$
\phi(\rho \theta(Y)) \ll \frac{A}{\varepsilon} \theta(Y) \sum_{q=1}^{+\infty}(\rho R \varepsilon)^{q}=\frac{A R \rho}{1-\varepsilon \rho R} \theta(Y)
$$

for all $\varepsilon>0$ such that $\varepsilon \rho R<1$.

Proof of Theorem 2.2. From the given assumptions on the vector fields $X_{j}$, it is sufficient to prove the analytic estimate for $\left\|\partial_{t_{k}}^{b} u\right\|_{\mu}$ for $k=1, \ldots, m$ and for every $b \geq 1$.

We fix $k$ and denote, for simplicity, $t=t_{k}$ and $T=\partial_{t}=\partial_{t_{k}}$. Then we define

$$
[u]_{t, r}=\sup _{0<q \leq r} \frac{\left\|T^{q} u\right\|_{\mu}}{M_{q}}
$$

and prove by induction on $r \geq 1$ that there exist $\varepsilon, M>0$ such that for all $r \geq 1$,

$$
[u]_{t, r} \leq M .
$$

We claim that we can take

$$
M=\max \left\{1, \frac{4}{c} \max _{1 \leq q \leq 3}\left\|T^{q} u\right\|_{\mu}\right\},
$$

whereas $\varepsilon$ will be chosen in the following.

For $p=1,2,3$ we clearly have that $[u]_{t, p} \leq M$ for $\varepsilon$ small enough. Assume that (12) is satisfied for all $3 \leq r<b$ and let us prove it for $r=b$ (the above request $b \geq 3$ will be understood in the following).

By the a-priori estimate (4) we have that

$$
\begin{aligned}
\left\|T^{b} u\right\|_{\mu} & =\sum_{j, k=1}^{n^{\prime}}\left\|X_{j} X_{k} T^{b} u\right\|_{\mu}+\sum_{j=1}^{n^{\prime}}\left\|X_{j} T^{b} u\right\|_{\mu}+\left\|T^{b} u\right\|_{\mu+\delta} \\
& \leq C\left(\left\|P T^{b} u\right\|_{\mu}+\left\|T^{b} u\right\|_{\mu}\right) .
\end{aligned}
$$

For every $\varepsilon_{1}, \delta_{1}>0$ we can find a positive constant $C_{\varepsilon_{1}, \delta_{1}}>0$ such that

$$
\left\|T^{b} u\right\|_{\mu} \leq \varepsilon_{1}\left\|T^{b} u\right\|_{\mu+\delta}+C_{\varepsilon_{1}, \delta_{1}}\left\|T^{b} u\right\|_{\mu-\delta_{1}} .
$$

Moreover

$$
\left\|P T^{b} u\right\|_{\mu} \leq\left\|\left[P, T^{b}\right] u\right\|_{\mu}+\left\|T^{b} P u\right\|_{\mu} .
$$

Since $\varepsilon_{1}\left\|T^{b} u\right\|_{\mu+\delta}$ will be absorbed in the left-hand side of (14), $\left\|T^{b} u\right\|_{\mu-\delta_{1}}$ will be estimated by induction and $\left\|T^{b} P u\right\|_{\mu}$ will not give any problems because of the 
analyticity of $f=P u$, we first estimate $\left\|\left[P, T^{b}\right] u\right\|_{\mu}$. To this aim we compute

$\left[T^{b}, P\right]=T^{b} P-P T^{b}$

$$
\begin{aligned}
= & \sum_{j, k=1}^{n^{\prime}} \sum_{b^{\prime}=1}^{b}\left(\begin{array}{c}
b \\
b^{\prime}
\end{array}\right) T^{b^{\prime}}\left(a_{j k}(u(t, x))\right) X_{j} X_{k} T^{b-b^{\prime}} \\
& +\sum_{j=1}^{n^{\prime}} \sum_{b^{\prime}=1}^{b}\left(\begin{array}{c}
b \\
b^{\prime}
\end{array}\right) T^{b^{\prime}}\left(b_{j}(u(t, x))\right) X_{j} T^{b-b^{\prime}}+\sum_{b^{\prime}=1}^{b}\left(\begin{array}{c}
b \\
b^{\prime}
\end{array}\right) T^{b^{\prime}}(c(u(t, x))) T^{b-b^{\prime}}
\end{aligned}
$$

since the $X_{j}$ 's do not depend on the variable $t$.

Let us denote by $a \circ u$ the generic coefficient $a_{j k}(u(t, x))$ or $b_{j}(u(t, x))$ or $c(u(t, x))$, and write $\left(X^{2}\right)$ for the generic term of the form 1 , or $X_{j}$ or $X_{j} X_{k}$. Then we estimate

$$
\begin{aligned}
\left\|\sum_{b^{\prime}=1}^{b}\left(\begin{array}{c}
b \\
b^{\prime}
\end{array}\right) T^{b^{\prime}}(a \circ u)\left(X^{2}\right) T^{b-b^{\prime}} u\right\|_{\mu} \leq & \Lambda\left\|T^{b}(a \circ u)\right\|_{\mu} \cdot\left\|\left(X^{2}\right) u\right\|_{\mu} \\
& +\Lambda \sum_{b^{\prime}=1}^{b-1}\left(\begin{array}{c}
b \\
b^{\prime}
\end{array}\right)\left\|T^{b^{\prime}}(a \circ u)\right\|_{\mu} \cdot\left\|\left(X^{2}\right) T^{b-b^{\prime}} u\right\|_{\mu} .
\end{aligned}
$$

It can be easily proved by induction on $p \geq 1$ that the derivative $T^{p}$ of the composite function $a(u(t, x))$ can be written as

$$
\begin{aligned}
T^{p}(a \circ u) & =\sum_{\substack{r_{i} \in \mathbb{N} \backslash\{0\} \\
r_{1}+\ldots+r_{q}=p}} C_{q, r} a^{(q)}(u) \partial_{t}^{r_{1}} u \cdots \partial_{t}^{r_{q}} u \\
& =a^{\prime}(u) T^{p} u+\sum_{\substack{r_{1}+\ldots+r_{q}=p \\
0<r_{i}<p}} C_{q, r} a^{(q)}(u) \partial_{t}^{r_{1}} u \cdots \partial_{t}^{r_{q}} u
\end{aligned}
$$

for some $C_{q, r}>0$, and therefore

$$
\begin{aligned}
\left\|T^{b}(a \circ u)\right\|_{\mu} \leq & \left\|a^{\prime}(u) T^{b} u\right\|_{\mu}+\sum_{\substack{r_{1}+\ldots+r_{q}=b \\
0<r_{i}<b}} C_{q, r} \Lambda^{q}\left\|a^{(q)}(u)\right\|_{\mu}\left\|\partial_{t}^{r_{1}} u\right\|_{\mu} \cdots\left\|\partial_{t}^{r_{q}} u\right\|_{\mu} \\
\leq & \Lambda\left\|a^{\prime}(u)\right\|_{\mu} \cdot\left\|T^{b} u\right\|_{\mu} \\
& +\sum_{\substack{r_{1}+\ldots+r_{q}=b \\
0<r_{i}<b}} C_{q, r}\left\|a^{(q)}(u)\right\|_{\mu}\left(\Lambda[u]_{t, b-1}\right)^{q} \partial_{t}^{r_{1}} \theta(0) \cdots \partial_{t}^{r_{q}} \theta(0) \\
(19) \quad &
\end{aligned}
$$

since $\left\|\partial_{t}^{r_{h}} u\right\|_{\mu} \leq[u]_{t, b-1} M_{r_{h}}=[u]_{t, b-1} \partial_{t}^{r_{h}} \theta(0)$ for $1 \leq r_{h} \leq b-1, h=1, \ldots, q$, where $\theta(Y)$ is the formal power series defined in (8).

With the choice made for $A, R>0$ in (9), we have that $\left\|a^{(q)}(u)\right\|_{\mu} \leq A R^{q} q$ ! and hence, substituting in (19),

$$
\begin{aligned}
\left\|T^{b}(a \circ u)\right\|_{\mu} \leq & \Lambda A R\left\|T^{b} u\right\|_{\mu} \\
& +\sum_{\substack{r_{1}+\ldots+r_{q}=b \\
0<r_{i}<b}} C_{q, r} A R^{q} q !\left(\Lambda[u]_{t, b-1}\right)^{q} \partial_{t}^{r_{1}} \theta(0) \cdots \partial_{t}^{r_{q}} \theta(0) .
\end{aligned}
$$


Let us now remark that, for $\phi$ given by (10) and $\rho>0$,

$$
\begin{aligned}
T^{b}(\phi(\rho \theta)) & =\sum_{\substack{r_{1}+\ldots+r_{q}=b \\
r_{i}>0}} C_{q, r} \phi^{(q)}(\rho \theta) \rho^{q} \partial_{t}^{r_{1}} \theta \cdots \partial_{t}^{r_{q}} \theta \\
& =\phi^{\prime}(\rho \theta) \rho \partial_{t}^{b} \theta+\sum_{\substack{r_{1}+\ldots+r_{q}=b \\
0<r_{i}<b}} C_{q, r} \phi^{(q)}(\rho \theta) \rho^{q} \partial_{t}^{r_{1}} \theta \cdots \partial_{t}^{r_{q}} \theta
\end{aligned}
$$

and therefore

$$
\left.T^{b}(\phi(\rho \theta(Y)))\right|_{Y=0}=A R \rho M_{b}+\sum_{\substack{r_{1}+\ldots+r_{q}=b \\ 0<r_{i}<b}} C_{q, r} A R^{q} q ! \rho^{q} \partial_{t}^{r_{1}} \theta(0) \cdots \partial_{t}^{r_{q}} \theta(0)
$$

since $\partial^{\alpha} \theta(0)=M_{|\alpha|}$ and $\phi^{(q)}(0)=A R^{q} q !$.

Substituting in (20) with $\rho=\Lambda[u]_{t, b-1}$,

$$
\begin{aligned}
\left\|T^{b}(a \circ u)\right\|_{\mu} \leq & \Lambda A R\left\|T^{b} u\right\|_{\mu}+\left.T^{b}\left(\phi\left(\Lambda[u]_{t, b-1} \theta(Y)\right)\right)\right|_{Y=0} \\
& -\Lambda A R[u]_{t, b-1} M_{b} .
\end{aligned}
$$

From (11) we deduce that

$$
T^{b}(\phi(\rho \theta(Y))) \ll \frac{A R \rho}{1-\varepsilon \rho R} T^{b} \theta(Y) \quad \text { if } \varepsilon \rho R<1,
$$

and hence

$$
\left.T^{b}\left(\phi\left(\Lambda[u]_{t, b-1} \theta(Y)\right)\right)\right|_{Y=0} \leq \frac{A R \Lambda[u]_{t, b-1}}{1-\varepsilon R \Lambda[u]_{t, b-1}} M_{b}
$$

if $\varepsilon R \Lambda[u]_{t, b-1}<1$.

By the inductive assumption we can take $\varepsilon=\varepsilon_{o} /(M R \Lambda)$, with $0<\varepsilon_{o}<1$ to be chosen in the following, so that

$$
\varepsilon R \Lambda[u]_{t, b-1} \leq \frac{\varepsilon_{o}}{M R \Lambda} R \Lambda M=\varepsilon_{o}<1 .
$$

We thus obtain from (21) and (15) that

$$
\begin{aligned}
\left\|T^{b}(a \circ u)\right\|_{\mu} \leq & \Lambda A R\left(\varepsilon_{1}\left\|T^{b} u\right\|_{\mu+\delta}+C_{\varepsilon_{1}, \delta_{1}}\left\|T^{b} u\right\|_{\mu-\delta_{1}}\right)+\frac{\Lambda A R}{1-\varepsilon_{o}}[u]_{t, b-1} M_{b} \\
& -\Lambda A R[u]_{t, b-1} M_{b} \\
(22) & \varepsilon_{1} \Lambda A R\left\|T^{b} u\right\|_{\mu+\delta}+\Lambda A R C_{\varepsilon_{1}, \delta_{1}}\left\|T^{b} u\right\|_{\mu-\delta_{1}}+\frac{\varepsilon_{o}}{1-\varepsilon_{o}} \Lambda A R[u]_{t, b-1} M_{b} .
\end{aligned}
$$

This estimate will be substituted in (18). In a similar way we obtain the following estimates for $1 \leq b^{\prime} \leq b-1$ :

$$
\begin{aligned}
\left\|T^{b^{\prime}}(a \circ u)\right\|_{\mu} & \leq \sum_{\substack{r_{1}+\ldots+r_{q}=b^{\prime} \leq b-1 \\
r_{i}>0}} C_{q, r} A R^{q} q !\left(\Lambda[u]_{t, b-1}\right)^{q} \partial_{t}^{r_{1}} \theta(0) \cdots \partial_{t}^{r_{q}} \theta(0) \\
& =\left.T^{b^{\prime}}\left(\phi\left(\Lambda[u]_{t, b-1} \theta(Y)\right)\right)\right|_{Y=0} \leq \frac{\Lambda A R}{1-\varepsilon_{o}}[u]_{t, b-1} M_{b^{\prime}}
\end{aligned}
$$


Substituting (22) and (23) in (18),

$$
\begin{aligned}
& \left\|\sum_{b^{\prime}=1}^{b}\left(\begin{array}{c}
b \\
b^{\prime}
\end{array}\right) T^{b^{\prime}}(a \circ u)\left(X^{2}\right) T^{b-b^{\prime}} u\right\|_{\mu} \leq \Lambda\left\|\left(X^{2}\right) u\right\|_{\mu}\left\{\varepsilon_{1} \Lambda A R\left\|T^{b} u\right\|_{\mu+\delta}\right. \\
& \left.\quad+\Lambda A R C_{\varepsilon_{1}, \delta_{1}}\left\|T^{b} u\right\|_{\mu-\delta_{1}}+\frac{\varepsilon_{o}}{1-\varepsilon_{o}} \Lambda A R[u]_{t, b-1} M_{b}\right\} \\
& \quad+\Lambda \sum_{b^{\prime}=1}^{b-1}\left(\begin{array}{c}
b \\
b^{\prime}
\end{array}\right) \frac{\Lambda A R}{1-\varepsilon_{o}}[u]_{t, b-1} M_{b^{\prime}}\left\|\left(X^{2}\right) T^{b-b^{\prime}} u\right\|_{\mu} .
\end{aligned}
$$

Let us set $m=\|\| u \|_{\mu}$ (so that $\left\|\left(X^{2}\right) u\right\|_{\mu} \leq m$ ), and estimate the norms $\left\|T^{b} u\right\|_{\mu-\delta_{1}}$ and $\left\|\left(X^{2}\right) T^{b-b^{\prime}} u\right\|_{\mu}$ :

$$
\begin{aligned}
& \left\|T^{b} u\right\|_{\mu-\delta_{1}} \leq\left\|T^{b-1} u\right\|_{\mu+\delta} \leq[u]_{t, b-1} M_{b-1} \quad \text { if } \delta_{1} \geq 1-\delta, \delta_{1}>0 \\
& \left\|\left(X^{2}\right) T^{b-b^{\prime}} u\right\|_{\mu} \leq\left\|T^{b-b^{\prime}} u\right\|_{\mu} \leq[u]_{t, b-1} M_{b-b^{\prime}} \quad \text { since } b^{\prime} \geq 1
\end{aligned}
$$

Then, from (24) and the inductive assumption,

$$
\begin{aligned}
\left\|\sum_{b^{\prime}=1}^{b}\left(\begin{array}{c}
b \\
b^{\prime}
\end{array}\right) T^{b^{\prime}}(a \circ u)\left(X^{2}\right) T^{b-b^{\prime}} u\right\|_{\mu} \leq \varepsilon_{1} m \Lambda^{2} A R\left\|T^{b} u\right\|_{\mu+\delta}+m \Lambda^{2} A R C_{\varepsilon_{1}, \delta_{1}} M M_{b-1} \\
\quad+\frac{\varepsilon_{o}}{1-\varepsilon_{o}} m \Lambda^{2} A R M M_{b}+\frac{\Lambda^{2} A R}{1-\varepsilon_{o}} M^{2} \sum_{b^{\prime}=1}^{b-1}\left(\begin{array}{c}
b \\
b^{\prime}
\end{array}\right) M_{b^{\prime}} M_{b-b^{\prime}} \\
\leq \varepsilon_{1} m \Lambda^{2} A R\left\|T^{b} u\right\|_{\mu+\delta}+m \Lambda^{2} A R C_{\varepsilon_{1}, \delta_{1}} M \varepsilon M_{b} \\
\quad+\frac{\varepsilon_{o}}{1-\varepsilon_{o}} m \Lambda^{2} A R M M_{b}+\frac{\Lambda^{2} A R}{1-\varepsilon_{o}} M^{2} \varepsilon M_{b}
\end{aligned}
$$

because of (17) and of the estimate $M_{b-1} \leq \varepsilon M_{b}$ for $b \geq 3$. (Here is the only reason why we start with $r=3$ in the induction.)

By the choice of $\varepsilon=\varepsilon_{o} /(M R \Lambda)$ we have

$$
\begin{aligned}
& \left\|\sum_{b^{\prime}=1}^{b}\left(\begin{array}{c}
b \\
b^{\prime}
\end{array}\right) T^{b^{\prime}}(a \circ u)\left(X^{2}\right) T^{b-b^{\prime}} u\right\|_{\mu} \leq \varepsilon_{1} m \Lambda^{2} A R\left\|T^{b} u\right\|_{\mu+\delta} \\
& \quad+\varepsilon_{o}\left(m \Lambda A C_{\varepsilon_{1}, \delta_{1}}+\frac{m \Lambda^{2} A R M}{1-\varepsilon_{o}}+\frac{\Lambda A M}{1-\varepsilon_{o}}\right) M_{b} .
\end{aligned}
$$

From (17) and (25) we finally obtain the desired estimate for $\left\|\left[P, T^{b}\right] u\right\|_{\mu}$ :

$$
\begin{aligned}
\left\|\left[P, T^{b}\right] u\right\|_{\mu} \leq & \varepsilon_{1} m \Lambda^{2} A R\left({n^{\prime}}^{2}+n^{\prime}+1\right)\left\|T^{b} u\right\|_{\mu+\delta} \\
& +\varepsilon_{o}\left(m \Lambda A C_{\varepsilon_{1}, \delta_{1}}+\frac{m \Lambda^{2} A R M}{1-\varepsilon_{o}}+\frac{\Lambda A M}{1-\varepsilon_{o}}\right)\left(n^{\prime 2}+n^{\prime}+1\right) M_{b} .
\end{aligned}
$$


Then, from (14), (15), (16) and (26),

$$
\begin{aligned}
\left\|T^{b} u\right\|_{\mu} \leq & C\left[\varepsilon_{1} m \Lambda^{2} A R\left({n^{\prime}}^{2}+n^{\prime}+1\right)\left\|T^{b} u\right\|_{\mu+\delta}\right. \\
& +\varepsilon_{o}\left(m \Lambda A C_{\varepsilon_{1}, \delta_{1}}+\frac{m \Lambda^{2} A R M}{1-\varepsilon_{o}}+\frac{\Lambda A M}{1-\varepsilon_{o}}\right)\left({n^{\prime}}^{2}+n^{\prime}+1\right) M_{b} \\
& \left.+B_{f}^{b+1} \frac{b !}{(b+1)^{2}}+\varepsilon_{1}\left\|T^{b} u\right\|_{\mu+\delta}+C_{\varepsilon_{1}, \delta_{1}}[u]_{t, b-1} M_{b-1}\right] \\
\leq & \varepsilon_{1}\left[C m \Lambda^{2} A R\left(n^{\prime 2}+n^{\prime}+1\right)+C\right] \cdot\left\|T^{b} u\right\|_{\mu+\delta} \\
& +\varepsilon_{o} C M\left(m \Lambda A C_{\varepsilon_{1}, \delta_{1}}+\frac{m \Lambda^{2} A R}{1-\varepsilon_{o}}+\frac{\Lambda A}{1-\varepsilon_{o}}\right)\left(n^{\prime 2}+n^{\prime}+1\right) M_{b} \\
& +B_{f}^{b+1} \frac{b !}{(b+1)^{2}}+C C_{\varepsilon_{1}, \delta_{1}} M \frac{\varepsilon_{o}}{M R \Lambda} M_{b},
\end{aligned}
$$

where $B_{f}>0$ is given by the analyticity of $f$.

We now choose $0<\varepsilon_{1}<1$ with

$$
A_{\varepsilon_{1}}=\varepsilon_{1}\left[C m \Lambda^{2} A R\left(n^{\prime 2}+n^{\prime}+1\right)+C\right]<1
$$

and then $0<\varepsilon_{o}<1$ sufficiently small so that, for $b \geq 3$,

$$
\frac{B_{f}^{b+1}}{A_{\varepsilon_{1}}} \frac{b !}{(b+1)^{2}} \leq \frac{1}{2} M_{b}=\frac{c}{2} \frac{\varepsilon_{o}^{1-b}}{(M R \Lambda)^{1-b}} \frac{b !}{(b+1)^{2}}
$$

and

$$
\varepsilon_{o} \frac{C}{A_{\varepsilon_{1}}}\left[\left(m \Lambda A C_{\varepsilon_{1}, \delta_{1}}+\frac{m \Lambda^{2} A R}{1-\varepsilon_{o}}+\frac{\Lambda A}{1-\varepsilon_{o}}\right)\left(n^{\prime 2}+n^{\prime}+1\right)+\frac{C_{\varepsilon_{1}, \delta_{1}}}{R \Lambda}\right] \leq \frac{1}{2} .
$$

With such choices we finally have that $\left\|T^{b} u \mid\right\|_{\mu} \leq M M_{b}$ and hence, by the inductive assumption,

$$
[u]_{t, b}=\sup _{0<q \leq b} \frac{\left\|T^{q} u\right\|_{\mu}}{M_{q}}=\max \left\{\sup _{0<q<b} \frac{\left\|T^{q} u\right\|_{\mu}}{M_{q}}, \frac{\left\|T^{b} u\right\|_{\mu}}{M_{b}}\right\} \leq M .
$$

The theorem is therefore proved.

\section{The Case of Coefficients also Depending on $(t, x) \in \mathbb{T}^{m} \times \mathbb{T}^{n}$}

Let us now consider the case in which the operator $P$ has real analytic complex valued coefficients which depend also on $(t, x) \in \mathbb{T}^{m} \times \mathbb{T}^{n} \simeq \mathbb{T}^{N}$ :

$$
\begin{aligned}
& P=P(t, x, u, D) \\
& =\sum_{j, k=1}^{n^{\prime}} a_{j k}(t, x, u(t, x)) X_{j} X_{k}+\sum_{j=1}^{n^{\prime}} b_{j}(t, x, u(t, x)) X_{j}+X_{0}+c(t, x, u(t, x)),
\end{aligned}
$$

where the real valued rigid vector fields $\left\{X_{j}\right\}_{0 \leq j \leq n^{\prime}}$ are defined as in (2) and satisfy the same assumptions as in $₫ 2$

Then we can prove the analogue of Theorem 2.2.

Theorem 3.1. Let $P$ be the operator defined in (27) and assume that the vector fields $\left\{X_{j}\right\}_{j=0, \ldots, n^{\prime}}$ are rigid and that for every fixed $x \in \mathbb{T}^{n}$ the $\left\{X_{j}^{\prime}\right\}_{j=1, \ldots, n^{\prime}}$ span $T_{x}\left(\mathbb{T}^{n}\right)$. 
Assume moreover that $u \in C^{\infty}\left(\mathbb{T}^{N}\right)$ is a solution of the equation

$$
P(t, x, u, D) u=f,
$$

for some $f \in A\left(\mathbb{T}^{N}\right)$ and that the a-priori estimate (4) is satisfied. Then also $u \in A\left(\mathbb{T}^{N}\right)$.

Proof. It is analogous to that of Theorem 2.2, and therefore we give here only the sketch of it.

Following the same outline as in the proof of Theorem 2.2 we replace (9) and (10) defining $A, R>1$ and the formal power series $\phi$ by the following formulas:

$$
\begin{aligned}
& \sum_{i, j=1}^{n^{\prime}}\left\|\partial_{t}^{r} \partial_{u}^{q} a_{i j}\right\|_{H^{\mu}\left(\mathbb{T}^{N} \times u\left(\mathbb{T}^{N}\right)\right)}+\sum_{j=1}^{n^{\prime}}\left\|\partial_{t}^{r} \partial_{u}^{q} b_{j}\right\|_{H^{\mu}\left(\mathbb{T}^{N} \times u\left(\mathbb{T}^{N}\right)\right)} \\
& +\left\|\partial_{t}^{r} \partial_{u}^{q} c\right\|_{H^{\mu}\left(\mathbb{T}^{N} \times u\left(\mathbb{T}^{N}\right)\right)} \leq A R^{r+q}(r+q) ! \quad \forall r, q \geq 0 \\
& \phi(w)=\sum_{q=1}^{+\infty} A(2 R)^{q} w^{q} \quad \text { for } w \in \mathbb{R},
\end{aligned}
$$

so that we have (11) with $\bar{R}=2 R$ instead of $R$. We then set

$$
M=\max \left\{1, \frac{4}{c} \max _{1 \leq q \leq 3}\left\|T^{q} u\right\|_{\mu}, 2 A\right\}
$$

instead of the choice made for $M$ in (13).

Following the proof of Theorem [2.2, we must estimate the $H^{\mu}\left(\mathbb{T}^{N}\right)$-norm of $T^{b} a(t, x, u(t, x))$. To this aim we first recall the following formula, which can be easily proved by induction on $p \geq 1$ :

$$
\begin{aligned}
& \partial_{t}^{p} a(t, x, u(t, x))=\left(\partial_{t}^{p} a\right)(t, x, u(t, x)) \\
& +\sum_{s=1}^{p}\left(\begin{array}{c}
p \\
s
\end{array}\right) \sum_{\substack{r_{1}+\ldots+r_{q}=s \\
r_{i}>0}} C_{q, r}\left(\partial_{u}^{q} \partial_{t}^{p-s} a\right)(t, x, u(t, x)) \partial_{t}^{r_{1}} u(t, x) \cdots \partial_{t}^{r_{q}} u(t, x)
\end{aligned}
$$

for some $C_{q, r}>0$. Then

$$
\begin{aligned}
& T^{b} a(t, x, u(t, x))=\left(\partial_{t}^{b} a\right)(t, x, u(t, x))+\left.\partial_{t}^{b} a(\tau, x, u(t, x))\right|_{\tau=t} \\
& \quad+\sum_{s=1}^{b-1}\left(\begin{array}{l}
b \\
s
\end{array}\right) \sum_{\substack{r_{1}+\ldots+r_{q}=s \\
r_{i}>0}} C_{q, r}\left(\partial_{u}^{q} \partial_{t}^{b-s} a\right)(t, x, u(t, x)) \partial_{t}^{r_{1}} u(t, x) \cdots \partial_{t}^{r_{q}} u(t, x),
\end{aligned}
$$

for $b \geq 3$. Let us denote by $a \circ u$ the composite function $a(t, x, u(t, x))$. The first term on the right-hand side of (29) is then easily estimated by

$$
\left\|\partial_{t}^{b} a \circ u\right\|_{\mu} \leq A R^{b} b ! \leq \frac{M}{2} M_{b}
$$

if $0<\varepsilon \leq \bar{\varepsilon}$ with $\bar{\varepsilon}$ small enough in order that

$$
\bar{R}^{p} p ! \leq M_{p}=\varepsilon^{1-p} c \frac{p !}{(p+1)^{2}} \quad \forall p \geq 2 .
$$

The second term on the right-hand side of (29) is estimated as in (22) with $\bar{R}=2 R$ instead of $R$ and $0<\bar{\varepsilon}_{o}<\min \{1, \bar{\varepsilon} M \bar{R} \Lambda\}$ instead of $\varepsilon_{o}$, taking $\varepsilon=\bar{\varepsilon}_{o} /(M \bar{R} \Lambda)$. 
We have to estimate the third term of the right-hand side of (29). To this aim we first recall that $(k+j) ! \leq 2^{k+j} k ! j$ ! for all $k, j \in \mathbb{N}$, and hence

$$
\left\|\partial_{u}^{q} \partial_{t}^{b-s} a \circ u\right\|_{\mu} \leq A R^{q+b-s}(q+b-s) ! \leq A \bar{R}^{q+b-s} q !(b-s) ! .
$$

Therefore

$$
\begin{aligned}
& \left\|\sum_{s=1}^{b-1}\left(\begin{array}{l}
b \\
s
\end{array}\right) \sum_{\substack{r_{1}+\ldots+r_{q}=s \\
r_{i}>0}} C_{q, r} \partial_{u}^{q} \partial_{t}^{b-s} a \circ u \partial_{t}^{r_{1}} u \cdots \partial_{t}^{r_{q}} u\right\|_{\mu} \\
\leq & \sum_{s=1}^{b-1}\left(\begin{array}{l}
b \\
s
\end{array}\right) \bar{R}^{b-s}(b-s) ! \sum_{\substack{r_{1}+\ldots+r_{q}=s \\
r_{i}>0}} C_{q, r} A \bar{R}^{q} q !\left(\Lambda[u]_{t, b-1}\right)^{q} \partial_{t}^{r_{1}} \theta(0) \cdots \partial_{t}^{r_{q}} \theta(0) \\
\leq & \left.\bar{R} b T^{b-1} \phi\left(\Lambda[u]_{t, b-1} \theta(Y)\right)\right|_{Y=0}+\left.\sum_{s=1}^{b-2}\left(\begin{array}{l}
b \\
s
\end{array}\right) M_{b-s} T^{s} \phi\left(\Lambda[u]_{t, b-1} \theta(Y)\right)\right|_{Y=0} \\
\leq & \frac{A \bar{R} \Lambda}{1-\bar{\varepsilon}_{o}}[u]_{t, b-1}\left[\bar{R} b M_{b-1}+\sum_{s=1}^{b-2}\left(\begin{array}{l}
b \\
s
\end{array}\right) M_{b-s} M_{s}\right] \\
\leq & \frac{3 A \bar{R}^{2} \Lambda}{1-\bar{\varepsilon}_{o}}[u]_{t, b-1} \varepsilon M_{b},
\end{aligned}
$$

because of (30), of

$$
\left.T^{p} \phi\left(\Lambda[u]_{t, b-1} \theta(Y)\right)\right|_{Y=0} \leq \frac{A \bar{R} \Lambda}{1-\bar{\varepsilon}_{o}}[u]_{t, b-1} M_{p} \quad \forall p \geq 1,
$$

of (77) and of $b M_{b-1} \leq 2 \varepsilon M_{b}$ for $b \geq 3$.

The same arguments can be hold to estimate $\left\|T^{b^{\prime}} a \circ u\right\|_{\mu}$ for $1 \leq b^{\prime} \leq b-1$. We can thus proceed as in the proof of Theorem 2.2 to obtain the desired estimate (12).

\section{ACKNOWLEDGEMENTS}

The authors are grateful to Professor P. Popivanov for his suggestions about the subject of this paper and for his helpful remarks.

\section{REFERENCES}

[AM] S. Alinhac - G. Metivier, Propagation de l'analyticité des solutions de systèmes hyperboliques non-linéaires, Invent. Math., 75 (1984), pp. 189-204 MR 86f:35010

[B] J.M. Bony, Calcul symbolique et propagation des singularités pour les équations aux dérivées partielles non linéaires, Ann. Scient. Éc. Norm. Sup., $4^{e}$ série (1981), pp. 209-246 MR 84h:35177

[GPY] T. Gramchev - P. Popivanov - M. Yoshino, Global properties in spaces of generalized functions on the torus for second order differential operators with variable coefficients, Rend. Sem. Mat. Univ. Pol. Torino, vol. 51, n. 2 (1993) MR 95k:35047

[G] P. Guan, Regularity of a class of quasilinear degenerate elliptic equations, Advances in Mathematics, 132 (1997), pp. 24-45 MR 99a:35068

[RS] L.P. Rothschild - E.M. Stein, Hypoelliptic differential operators and nilpotent groups, Acta Math., 137 (1976), pp. 247-320 MR 55:9171

[T] D.S. Tartakoff, Global (and local) analyticity for second order operators constructed from rigid vector fields on products of tori, Trans. of A.M.S., Vol. 348, n. 7 (1996), pp. 2577-2583 MR 96i:35018 
[TZ] D.S. Tartakoff - L. Zanghirati, Analyticity of Solutions for Sums of Squares of Non-linear Vector Fields, to appear in Proc. A.M.S.

[X] C.J. Xu, Regularity of solutions of second order non-elliptic quasilinear partial differential equations, C.R. Acad. Sci. Paris, Sér. I, n. 8, t. 300 (1985), pp. 235-237 MR 86m:35073

Dipartimento di Matematica, Via Machiavelli n.35, 44100 Ferrara, Italy

E-mail address: boiti@dm.unife.it

Dipartimento di Matematica, Via Machiavelli n.35, 44100 Ferrara, Italy

E-mail address: zan@dns.unife.it 